\title{
Gingival Stimulation: An Important Metabolic Regulator?
}

\author{
Yildirim $\mathrm{A}^{1}$, Atmaca $\mathrm{H}^{* 2}$, Bedir $\mathrm{A}^{3}$ and Devrim $\mathrm{I}^{4}$
}

${ }^{1}$ Department of Internal Medicine, Faculty of Medicine, Ondokuz Mayis University, Samsun, Turkey

${ }^{2}$ Department of Endocrinology and Metabolism, Faculty of Medicine, Ondokuz Mayis University, Samsun, Turkey

${ }^{3}$ Department of Biochemistry, Faculty of Medicine, Ondokuz Mayis University, Samsun, Turkey

${ }^{4}$ Department of Periodontology, Faculty of Dentistry, Ondokuz Mayis University, Samsun, Turkey

*Corresponding author: Atmaca H, MD, Professor, Department of Endocrinology and Metabolism, Liv Hospital, Faculty of Medicine, Ondokuz Mayis University, Samsun, Turkey, Tel: 5355552198, E-mail: mhatmaca@gmail.com

Citation: Yildirim A, Atmaca H, Bedir A, Devrim I (2017) Gingival Stimulation: An Important Metabolic Regulator? J Obes Overweig 3(2): 204. doi: 10.15744/2455-7633.3.204

Received Date: October 12, 2017 Accepted Date: November 28, 2017 Published Date: November 30, 2017

\begin{abstract}
Objective: This study aimed to determine whether a relationship exists between gingival stimulation and the levels of leptin, ghrelin, insulin and glucose, which are important regulators of energy homeostasis.

Materials and Methods: Blood samples for ghrelin, leptin, glucose and insulin were taken from 15 male volunteers (mean age $25.5 \pm 2.3$ years; mean body mass index $24.4 \pm 2.79 \mathrm{~kg} / \mathrm{m}^{2}$ ), who did not brush their teeth for one day, after a $12 \mathrm{~h}$-long overnight fasting and before standard breakfast ( $0 \mathrm{~min})$ and thereafter at 30, 60, 120 and $180 \mathrm{~min}$ after breakfast. After tooth brushing after dinner and after a $12 \mathrm{~h}-\mathrm{long}$ overnight fasting, blood samples were taken again before standard breakfast $(0 \mathrm{~min})$ and then after at the same time points following tooth brushing.
\end{abstract}

Results: A significant reduction was found in the leptin levels measured at 0, 30, 60, 120 and 180 min after tooth brushing $(p<0.005)$. The ghrelin levels also declined at these time points but were significant at 0,30 and $120 \min (p<0.05)$. Despite the reduced insulin levels at 120 and 180 min after tooth brushing $(p<0.05)$, no significant change was observed in the glucose levels.

Conclusion: Mechanical stimulation of the gingiva by tooth brushing may play a role in appetite regulation by influencing the ghrelin, leptin and insulin signalling pathways.

Keywords: Appetite; Ghrelin; Gingival Stimulation; Insulin; Leptin; Tooth Brushing

List of abbreviations: BMI: Mean Body mass index; CNS: Central nervous system; HOMA: Homeostasis model assessment; GI: Gastrointestinal; GHS: Growth hormone secretagogue; MS: Metabolic syndrome; NPY: Neuropeptide Y

\section{Introduction}

Ghrelin and leptin regulate energy balance primarily through the hypothalamic neurons in the central nervous system (CNS). Ghrelin stimulates the activity of arcuate neuropeptide Y (NPY) neurons. However, the appetite-promoting NPY neurons are inhibited by leptin. Therefore, both function as mutual antagonists in the hypothalamus, which regulates feeding behaviour [1]. Insulin and glucose are also important co-regulators of energy homeostasis [1]. As a processing centre for appetite, the hypothalamus integrates signals from the brain, the peripheral circulation and the gastrointestinal (GI) tract to regulate energy intake and expenditure [2]. Neurons expressing these neuropeptides communicate with each other and with many peripheral signals, including nutrients such as glucose, GI peptide hormones such as ghrelin, and other hormones such as insulin and leptin, to influence appetite level and feeding [3]. Therefore, the GI tract is an important and active part of energy regulation. However, the stomach and intestine usually attract more attention than other parts of the GI tract in terms of appetite regulation [2]. The implication of the mouth in energy regulation has rarely been studied [1].

Sakata et al. showed that the mastication-induced activation of histamine neurons suppressed physiological food intake through the H1-receptor in the hypothalamic paraventricular nucleus and the ventromedial hypothalamus in rats [4]. Oral proprioceptive signals received at the mesencephalic trigeminal sensory nucleus (Me5) could modulate the hypothalamic histamine neurons through the ascending pathway from Me5 to the posterior hypothalamus [5]. Moreover, histamine neurons were found to be 
concordant with the leptin signalling system through a negative feedback loop. Leptin infusion elevated the turnover rate of neuronal histamine in the hypothalamus. Similar to mastication, whether gingival stimulation can affect the histaminergic neuron system should be elucidated.

The dental pulp and gingiva are highly vascularised connective tissues. Studies have shown that these tissues are active in terms of ghrelin and leptin production and are also a target of these hormones [6-10].

This study specifically investigated the possible role of tooth brushing as an initial part of energy regulation. This work is the first so far to evaluate leptin, ghrelin, insulin and glucose levels in relation to tooth brushing.

\section{Materials and Methods}

\section{Participants}

The study included 15 male volunteers (mean age: 25.5 \pm 2.3 years; age range: $23-31$ ), who were final-year medical students or research assistants at the Faculty of Medicine, Ondokuz Mayis University. The participants did not have any systemic diseases, experience recent weight loss, participate in any dieting behaviour, smoke, or receive periodontal treatment in the previous six months. As sex hormones may affect the leptin levels, the participants were chosen to be all male [11]. Moreover, to form a homogeneous group in terms of insulin sensitivity, the participants with similar lifestyles, who were either medical students or research assistants at the Faculty of Medicine, were recruited. The study protocol was approved by the local ethics committee of Ondokuz Mayis University. All subjects signed an informed written consent prior to participation.

\section{Protocol}

This study aimed to investigate the possible effects of tooth brushing on appetite hormones. The investigated hormones and glucose are known to be affected by fasting and eating, but the effect of gingival stimulation in the form of tooth brushing is unknown. If such an effect exists, then its onset and duration should be determined. This work was a physiological study, and we attempted to simulate a normal lifestyle in subjects during the study period. For this reason, the hormones were evaluated before and after tooth brushing and meals. Breakfast in this study contained approximately $430 \mathrm{Kcal}$ (56\% carbohydrates, $17 \%$ protein and $27 \%$ fat).

The long-term exposure of the gingiva to microbial dental plaque may cause gingival inflammation [12]. The lack of tooth brushing, which is known to accelerate plague formation and inflammation, could interfere with the study. Thus, the participants brushed their teeth regularly before the study. They were only allowed not to brush their teeth for one day at the beginning of the study. Blood samples were taken from the subjects who did not brush their teeth for one day, after a 12 h-long overnight fasting and before standard breakfast ( $0 \mathrm{~min}$ ) and thereafter at 30,60, 120 and $180 \mathrm{~min}$ after breakfast.

To simulate a normal life condition, the subjects were allowed to brush their teeth after the following dinner and breakfast. After tooth brushing following dinner and a $12 \mathrm{~h}$-long overnight fasting, blood samples were taken again before standard breakfast $(0$ min) and then after at 30, 60, 120 and 180 min following tooth brushing without toothpaste. Ghrelin, leptin, glucose and insulin levels were measured from the blood samples. The study scheme is illustrated in detail in Figure 1.

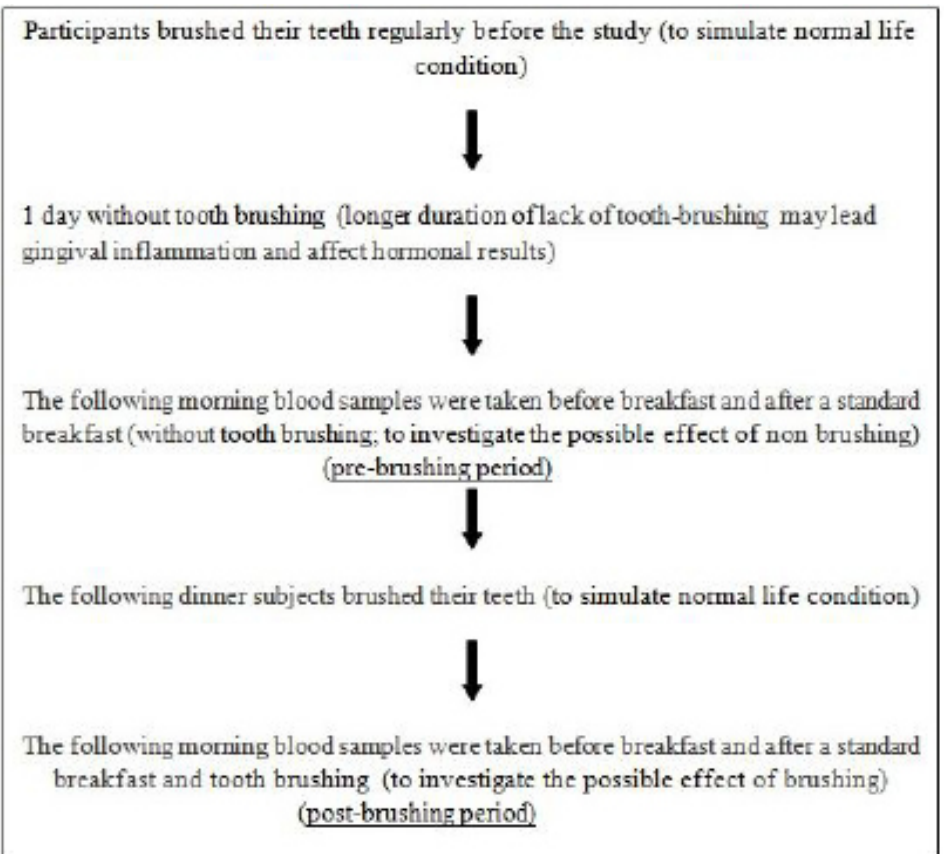

Figure 1: Leptin, ghrelin, insulin and glucose levels were evaluated before and after both tooth brushing and meals to simulate a normal lifestyle among the subjects during the study period 
The estimate of insulin resistance by the homeostasis model assessment (HOMA) score was calculated with the following formula: fasting serum insulin $(\mu \mathrm{U} / \mathrm{ml}) \times$ fasting serum glucose $(\mathrm{mmol} / \mathrm{L}) / 22.5)$ [13]. Leptin, ghrelin, glucose and insulin levels were expressed as the area under the curve (AUC) estimated by the trapezoidal rule. Each subject served as his own control for the effect of tooth brushing on serum hormones.

\section{Blood sampling and processing}

Venous blood samples were drawn into plain vacutainers and kept at room temperature until clotting. Serum samples were separated by centrifugation at 3000x g for $10 \mathrm{~min}$ and stored at $-80{ }^{\circ} \mathrm{C}$ until assay. Serum leptin and total ghrelin levels were measured by commercial ELISA kits (DIAsource Leptin-EASIA Kit, Belgium and RayBio Human/Mouse/Rat Ghrelin Enzyme Immunoassay Kit, Germany). The precision performance of ELISA kits was evaluated with low and high levels of the control material included. Fasting blood glucose and insulin levels were measured on a Cobas 8000 modular analyser (Roche Diagnostics, Indianapolis, USA) by dedicated reagents.

\section{Statistical analysis}

Data were analysed by SPSS 15.0 software. Normality test was performed for continuous variables and parametric (paired-t test and Pearson correlation analysis) and nonparametric tests (Wilcoxon signed rank test, Friedman analysis of variance and Bonferroni adjusted Wilcoxon signed rank test) were used to evaluate the data according to whether they were distributed normally or not. A value of $\mathrm{p}<0.05$ was accepted as statistically significant for the paired-t test, Wilcoxon signed rank test and Friedman analysis of variance, and a value of $\mathrm{p}<0.01$ was considered statistically significant for the Bonferroni-adjusted Wilcoxon signed rank test. Data distributed normally were presented as the mean $\pm \mathrm{SD}$, whereas those that were not distributed normally were presented as the median (minimum-maximum).

\section{Results}

The mean body mass index (BMI) of the participants was $24.4 \pm 2.79 \mathrm{~kg} / \mathrm{m}^{2}$ (range: 19-29). A significant reduction was found in the leptin levels measured at $0(p=0.01), 30(p=0.002), 60(p=0.001), 120(p=0.004)$ and $180(p=0.001)$ min after tooth brushing (Figure 2). The ghrelin levels also declined at these time points, which were significant at $0(p=0.032), 30(p=0.009)$ and $120 \mathrm{~min}$ $(p=0.011)$ (Figure 3). Similarly, both the $\mathrm{AUC}_{\text {leptin }}(p=0.001)$ and $\mathrm{AUC}_{\text {ghrelin }}(p=0.006)$ levels were lower in the post-brushing period than in the pre-brushing period (Table 1). Despite the reduced insulin levels at $120(p=0.027)$ and $180(p=0.016)$ min after tooth brushing (Figure 4$)$, no significant change was observed in the glucose levels $(p>0.05)$ (Figure 5). Although the $\mathrm{AUC}_{\text {insulin }}$ levels declined in the post-brushing period, these differences were statistically insignificant $(p=0.053)$. Moreover, the AUC ${ }_{\text {glucose }}$ levels did not change between the pre and post- brushing periods $(p=0.828)$ (Table 1$)$. The HOMA scores did not differ between prebrushing $(0.87 \pm 0.73)$ and post-brushing $(1.35 \pm 1.40)$ periods $(p=0.281)$.

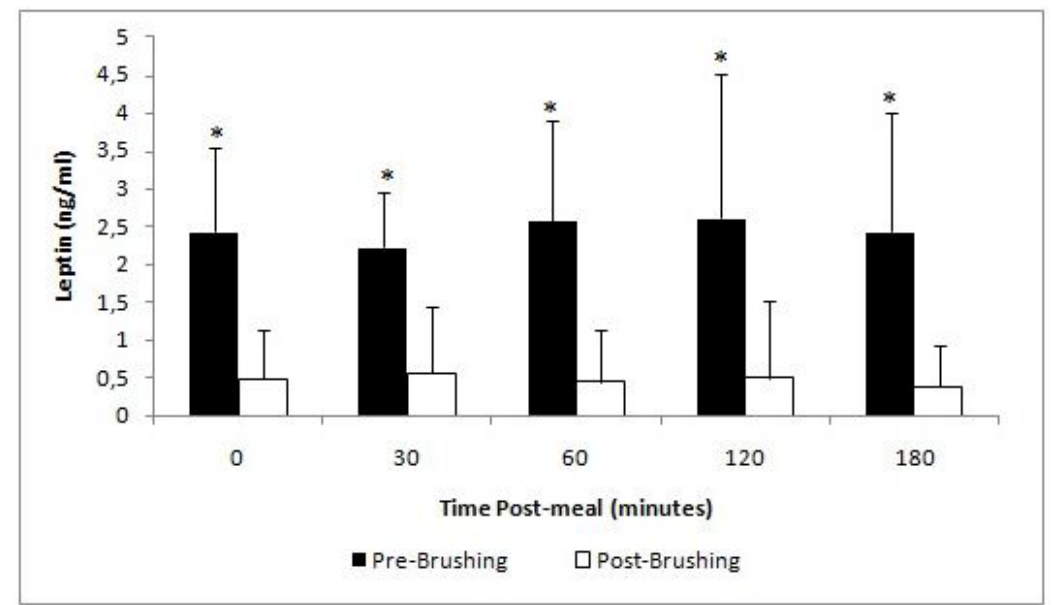

Figure 2: Mean $( \pm S D)$ leptin levels in the pre-brushing and post-brushing periods. A significant reduction was found in the leptin levels in the post-brushing period for all time points measured at $0(\mathrm{p}=0,001), 30(\mathrm{p}=0,002), 60(\mathrm{p}=0,001), 120(\mathrm{p}=0,004)$ and $180(\mathrm{p}=0,001)$ min

\begin{tabular}{|c|c|c|c|}
\hline & Pre-brushing & Post-brushing & P \\
\hline $\mathrm{AUC}_{\text {leptin }}(\mathrm{ng} / \mathrm{ml} \times 180 \mathrm{~min})$ & $447 \pm 198$ & $86 \pm 114$ & 0.001 \\
\hline $\mathrm{AUC}_{\text {ghrelin }}(\mathrm{ng} / \mathrm{ml} \times 180 \mathrm{~min})$ & $3228 \pm 955$ & $2556 \pm 506$ & 0.006 \\
\hline $\mathrm{AUC}_{\text {insulin }}(\mu \mathrm{U} / \mathrm{ml} \times 180 \mathrm{~min})$ & $3796 \pm 1915$ & $3226.4 \pm 1621.9$ & 0.053 \\
\hline $\mathrm{AUC}_{\text {glucose }}(\mathrm{mg} / \mathrm{dl} \times 180 \mathrm{~min})$ & $14777 \pm 1786$ & $14728.0 \pm 1555$ & 0.828 \\
\hline
\end{tabular}

Values are the mean \pm SD

Table 1: Area under the curve (AUC) values of leptin, ghrelin, insulin and glucose in the pre-brushing and post-brushing periods 


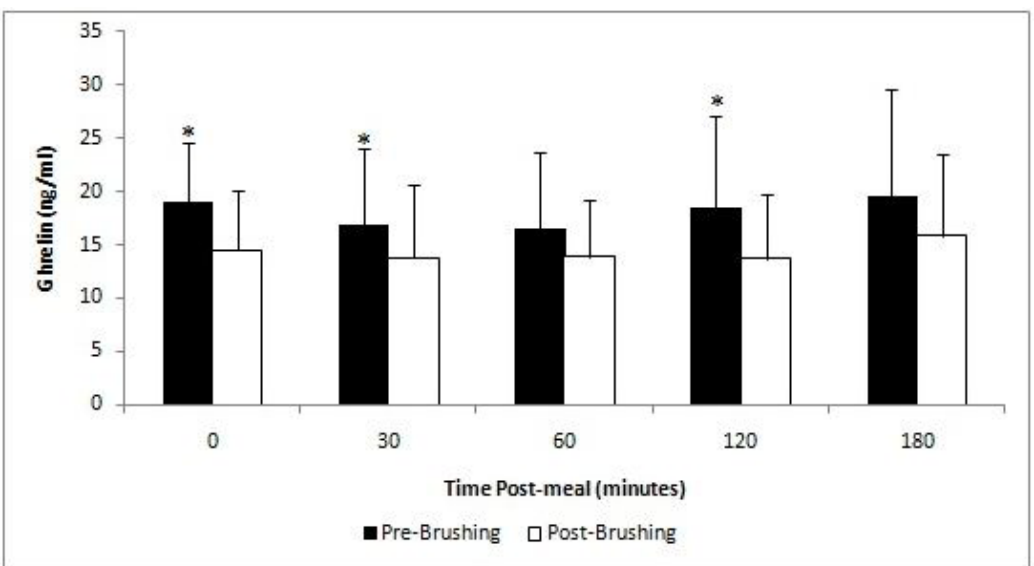

Figure 3: Mean $( \pm \mathrm{SD})$ ghrelin levels in the pre-brushing and post-brushing periods. The ghrelin levels declined in the post-brushing period and were significant at the time points of 0 $(\mathrm{p}=0.032), 30(\mathrm{p}=0.009)$ and $120(\mathrm{p}=0.011) \mathrm{min}$

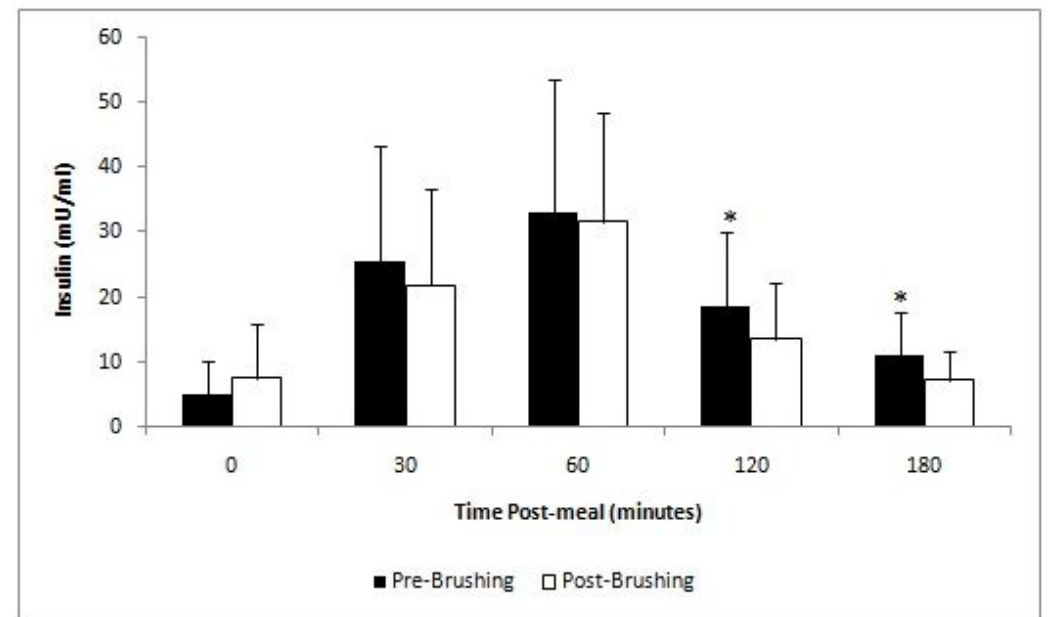

Figure 4: Mean $( \pm S D)$ insulin levels in the pre-brushing and post-brushing periods. The mean insulin levels declined in the post-brushing period and were significant at the time points of $120(\mathrm{p}=0.027)$ and $180(\mathrm{p}=0.016) \mathrm{min}$

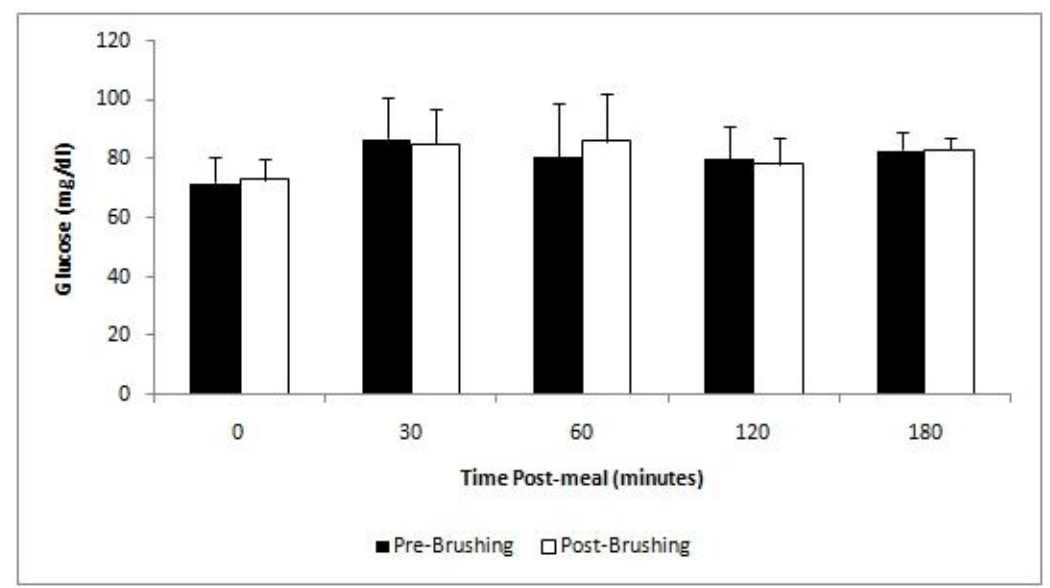

Figure 5: Mean $( \pm S D)$ glucose levels in the pre-brushing and post-brushing periods. The mean glucose levels did not change between the pre and post-brushing periods at any time point $(\mathrm{p}>0.05)$

\section{Discussion}

We found that gingival stimulation in the form of tooth brushing decreases the leptin, ghrelin and, partly, insulin levels in nonobese healthy adults males. The mechanism for these alterations is not clear.

Gingival tissues have been shown to produce both ghrelin [6] and leptin [7], and both plasma levels increased [8,9] in patients with chronic periodontitis. Gingival tissues also express leptin receptor as well as ghrelin mRNA and growth hormone secretagogue 
(GHS) receptors $[6,10]$. As demonstrated in this study, gingival stimulation leads to hormonal alterations, and it offers a new avenue for the investigation of the active participation of this tissue.

Ghrelin stimulates hunger, leptin promotes satiety, and both affect the insulin and glucose responses [14,15]. The implication of decreased leptin, ghrelin and insulin following tooth brushing in appetite control is not clear. Adiposity signals, such as insulin and leptin, are involved in long-term energy homeostasis, and ghrelin, which is a gut hormone, is implicated in the short-term regulation of meal ingestion [16]. Therefore, the effect of hormone level alterations on the energy homeostasis observed in this study is unusual.

Numerous studies have investigated the association of tooth brushing frequency with the risk of metabolic syndrome (MS). Regular tooth brushing has been shown to be associated with the decreased risk of type 2 diabetes mellitus, hypertension, dyslipidaemia and obesity, which are components of MS [17,18]. A low frequency of tooth brushing increases the incidence of periodontal disease [19]. Similar results were found on the association between periodontal diseases and MS [20-22]. However, the causality between them remains controversial.

One of the potential links between periodontal disease and MS is oxidative stress [23]. Oxidative stress, as a consequence of MS and/or periodontal disease, leads to inflammation and insulin resistance. The direction of the relationship between MS and periodontal disease cannot be determined currently because the majority of studies on this topic are cross-sectional [21]. The present study provides both a specific mechanism and a direction for this association. The evidence of reduced insulin levels after tooth brushing suggests the role of insulin sensitivity in the development of MS associated with the low frequency of tooth brushing. The finding of decreased leptin and ghrelin levels in parallel with the insulin level implicates the appetite hormones in this mechanism for the first time. Moreover, this study establishes evidence on the acute hormonal effect of tooth brushing.

The decrease in the ghrelin and leptin levels at 0 min after the pre-brushing period could also be caused by tooth brushing $12 \mathrm{~h}$ ago, as the $0 \mathrm{~min}$ values represent those before breakfast and tooth brushing. This finding is suggestive of the prolonged effect of tooth brushing. Another noteworthy result is about the insulin levels, which decreased following brushing at 120 and 180 min. Further blood samples after these time points showed the prolonged effect of tooth brushing on insulin secretion. Nevertheless, based on these findings, the prolonged effect of tooth brushing on ghrelin and leptin secretion was greater than that on insulin secretion. Despite the reduced insulin levels at 120 and $180 \mathrm{~min}$ after tooth brushing, no significant changes were observed in both glucose level and HOMA scores. However, the prolonged effects of tooth brushing on these hormones should be investigated by hormone profiles for at least $24 \mathrm{~h}$.

These hormones were evaluated before and after both tooth brushing and standard breakfast. Thus, we aimed to obtain the complex real effects of tooth brushing by simulating normal life conditions in this study. In an additional experiment, the effect of tooth brushing in the absence of calorie intake will emerge more precisely.

The participants of this study were males and were either medical students or research assistants at the Faculty of Medicine. Their age and BMI were close to each other, and their lifestyles were similar. Despite the limited number of the subjects, the homogeneity of this group makes the results of this study robust.

\section{Conclusion}

This preliminary study established that gingival stimulation by tooth brushing in non-obese young adult men resulted in the reduced secretion of ghrelin, leptin and insulin, which are involved in appetite and energy homeostasis. Further understanding of the nutrition physiology and metabolism can be obtained with similar studies to be conducted on slim and obese individuals. The role of such hormonal changes on appetite control should be elucidated with appropriately designed studies. Moreover, this study showed that tooth brushing is not only important for dental care but also for body care.

\section{Acknowledgment}

We would like to thank our department nurses, Gulay Gulsen and Almila Yildirim, and our secretary, Bayram Sanci, for their assistance during the tests. This study was supported by the Ondokuz Mayis University Scientific Research Funding (Project code no: 1904.13.027).

\section{Authorship Contributions}

Yildirim A designed and conducted the study as well as analysed and interpreted the data. Atmaca $\mathrm{H}$ designed the study, analysed and interpreted data and drafted the article. Sunter AT performed the statistical tests, interpreted the data and revised the article. Bedir A analysed and interpreted the data. Devrim I was responsible for the conception and design of the study.

\section{References}

1. Sun Y, Asnicar M, Smith RG (2007) Central and peripheral roles of ghrelin on glucose homeostasis. Neuroendocrinology 86: 215-28.

2. Neary NM, Goldstone AP, Bloom SR (2004) Appetite regulation: from the gut to the hypothalamus. Clin Endocrinol (Oxf) 60: 153-60.

3. Havel PJ (2001) Peripheral signals conveying metabolic information to the brain: short-term and long-term regulation of food intake and energy homeostasis. Exp Biol Med (Maywood) 226: 963-77. 
4. Sakata T, Yoshimatsu H, Masaki T, Tsuda K (2003) Anti-obesity actions of mastication driven by histamine neurons in rats. Exp Biol Med (Maywood) 2288: $1106-10$.

5. Ericson H, Blomqvist A, Köhler C (1991) Origin of neuronal inputs to the region of the tuberomammillary nucleus of the rat brain. J Comp Neurol 311: 45-64. 6. Ohta K, Laborde NJ, Kajiya M, Shin J, Zhu T, et al. (2011) Expression and posible immune-regulatory function of ghrelin in oral epithelium. J Dent Res 90: 1286-92.

7. Johnson RB, Serio FG (2001) Leptin within healthy and diseased human gingiva. J Periodontol 72: 1254-7.

8. Liu YY, Zhang X, Shi D, Cheng ZB, Meng HX (2013) Association between plasma leptin level and periodontal parameters in patients with aggressive periodontitis. Zhonghua Kou Qiang Yi Xue Za Zhi 48: 3-6.

9. Yılmaz G, Kırzıŏlu FY, Doğuç DK, Koçak H, Orhan H (2014) Ghrelin levels in chronic periodontitis patients. Odontology $102: 59-67$.

10. Ay ZY, Kırzıoğlu FY, Tonguç MO, Sütçü R, Kapucuoğlu N (2012) The gingiva contains leptin and leptin receptor in health and disease. Odontology 100: 222-31.

11. Margetic S, Gazzola C, Pegg GG, Hill RA (2002) Leptin: a review of its peripheral actions and interactions. Int J Obes Relat Metab Disord 26: 1407-33.

12. Axelsson P, Lindhe J (1981) Effect of controlled oral hygiene procedures on caries and periodontal disease in adults. Results after 6 years. J Clin Periodontol 8: $239-48$.

13. Bonora E, Targher G, Alberiche M, Bonadonna RC, Saggiani F, et al. (2000) Homeostasis model assessment closely mirrors the glucose clamp technique in the assessment of insulin sensitivity. Diabetes Care 23: 57-63.

14. Carlson JJ, Turpin AA, Wiebke G, Hunt SC, Adams TD (2009) Pre- and post- prandial appetite hormone levels in normal weight and severely obese women. Nutr Metab 6: 32.

15. Chabot F, Caron A, Laplante M, St-Pierre DH (2014) Interrelationships between ghrelin, insulin and glucose homeostasis: Physiological relevance. World J Diabetes 5: 328-41.

16. Suzuki K, Jayasena CN, Bloom SR (2012) Obesity and appetite control. Exp Diabetes Res 2012: 824305.

17. Kuwabara M, Motoki Y, Sato H, Fujii M, Ichiura K, et al. (2017) Low frequency of toothbrushing practices is an independent risk factor for diabetes mellitus in male and dyslipidemia in female: A large-scale, 5-year cohort study in Japan. J Cardiol 70: 107-12.

18. Kuwabara M, Motoki Y, Ichiura K, Fujii M, Inomata C, et al. (2016) Association between toothbrushing and risk factors for cardiovascular disease: a large-scale, cross-sectional Japanese study. BMJ Open 6: e009870.

19. Glickman I (1971) Periodontal disease. N Engl J Med 284: 1071-7.

20. Furuta M, Shimazaki Y, Takeshita T, Shibata Y, Akifusa S, et al. (2013) Gender differences in the association between metabolic syndrome and periodontal disease: the Hisayama Study. J Clin Periodontol 40: 743-52.

21. Lamster IB, Pagan M (2016) Periodontal disease and the metabolic syndrome. Int Dent J 67: 67-77.

22. Martens L, De Smet S, Yusof MY, Rajasekharan S (2017) Association between overweight/obesity and periodontal disease in children and adolescents: a systematic review and meta-analysis. Eur Arch Paediatr Dent 18: 69-82.

23. Bullon P, Morillo JM, Ramirez-Tortosa MC, Quiles JL, Newman HN, et al. (2009) Metabolic syndrome and periodontitis: is oxidative stress a common link? J Dent Res 88: 503-18. 